\title{
Multiphysics Modeling of Plasmonic Photothermal Heating Effects in Gold Nanoparticles and Nanoparticle Arrays
}

Santiago Manrique-Bedoya, ${ }^{\dagger}$ Mohammad Abdul-Moqueet, ${ }^{\ddagger}$ Priscilla Lopez, ${ }^{\ddagger}$ Tara Gray, ${ }^{\ddagger}$ Matthew Disiena, ${ }^{\ddagger}$ Sharon Kwee, $₫$ Liang Tang, "Lyle Hood ${ }^{\dagger}$ Yusheng Feng, ${ }^{\dagger}$ Nicolas Large, ${ }^{\ddagger}$ and Kathryn M. Mayer ${ }^{*, \ddagger}$

$\dagger$ Department of Mechanical Engineering; The University of Texas at San Antonio $\ddagger$ Department of Physics and Astronomy; The University of Texas at San Antonio IDepartment of Biomedical Engineering; The University of Texas at San Antonio

E-mail: kathryn.mayer@utsa.edu

\section{Supporting Information}

Optical absorbance by GNPs

Given the relationship between the electric and magnetic fields, where the ratio of the electric to magnetic fields equals the speed of light, ${ }^{1}$ it is possible to fully describe electromagnetic waves using one of the two wave equations. The wave equation for electric field, assuming there are no source charges, was used in COMSOL's setup as shown in Equation 1:

$$
\nabla \times \mu^{-1} \nabla \times \mathbf{E}-\mathrm{k}_{0}^{2} \varepsilon \mathbf{E}=0
$$

Accurate representation of light-particle interaction required proper input for both the wave 
and the material properties of the GNP. Firstly, a plane wave polarized in the y-direction and propagating in the z-direction was used as the background, or incident, electric field as shown below in Equation 2:

$$
\mathbf{E}_{\mathbf{b}}=\left\{0, \mathbf{E}_{\mathbf{0}} \mathrm{e}^{\left(-\mathrm{j} \frac{2 \pi \mathrm{n}}{\lambda} \mathrm{z}\right)}, 0\right\}
$$

Here, $\mathbf{E}_{\mathbf{0}}$ is the amplitude of the wave, $j$ is the imaginary unit number, $n$ is the refractive index of the medium, and $\lambda$ is the wavelength. Note that the amplitude of the wave $\left(\mathbf{E}_{0}\right)$ depends on the laser irradiance, or intensity $(I)$, as shown in Equation 3.

$$
\mathbf{E}_{\mathbf{0}}=\sqrt{\frac{2 \mathrm{I}}{\mathrm{cn} \varepsilon_{0}}}
$$

Where $c$ is the speed of light in vacuum, and $\varepsilon_{0}$ is the vacuum permittivity. Secondly, two material properties were required for the GNPs: Relative dielectric permittivity $\varepsilon_{r}$, and relative magnetic permeability $\mu_{r}$. For gold in the wavelength range of interest, the permittivity is both complex and wavelength dependent. Dielectric permittivity tabulated by Johnson and Christy ${ }^{2}$ was used over the entire wavelength range of interest. As gold does not exhibit magnetization under the presence of magnetic fields, we assumed a value of magnetic permeability very close to unity.

Solving Equation 1 using numerical methods requires truncation of the computational domain so that no significant artifacts are introduced into the computation. Due to the oscillatory behavior of the solutions found for wave equations, implementation of Dirichlet or Neumann boundary conditions would introduce boundary reflections to the computation. Therefore, a non-reflective boundary that could absorb the waves was implemented. ${ }^{3,4}$ This boundary, called a perfectly matched layer (PML), attenuates the incoming wave by inducing an exponential decay, thus preventing reflection of the wave at the boundaries. ${ }^{3,5-7}$

The formulation of the PML is analytical, so it is reflectionless as long as the exact wave equations are being solved. When using numerical solvers, the discretization of the domain caused the PML to not be perfectly absorbing, but the waves propagating through it are still being attenuated. To achieve a negligible effect of the reflected waves, a thickness of $\lambda / 2$ 
is sufficient. ${ }^{3}$

The computational domain was partitioned into smaller elements so that the discretized equations were solved using COMSOL's iterative solvers. The mesh setting was physics controlled, meaning that the software determined the most appropriate mesh distribution according to the geometry and input parameters, such as the thickness of the five layers in the PML, and the element distribution throughout the nanoparticle and the surrounding media domains. With an overall computational domain size around $1.6 \mu \mathrm{m}$, and the nanoparticle sizes ranging between 40 and $160 \mathrm{~nm}$, the built-in finer mesh element size (element sizes ranging from 4.3 to $59.3 \mathrm{~nm}$ ) was selected giving a total of more than $3 \times 10^{4}$ elements for the different nanoparticle shapes. The default solver selected for solving simulations using electromagnetic waves is the biconjugate gradient stabilized method (BiCGStab) which converges to find a solution that shows the electric and magnetic fields in the entire computational domain.

Heat transfer for GNPs in a homogenous environment (single GNPs and arrays)

The initial temperature of the system is $37^{\circ} \mathrm{C}$ so that it resembles the internal temperature of the human body; therefore, an assumption of this temperature being constant at a distance further away from the particle is in place. In this case, more than $6.4 \times 10^{4}$ elements were used to discretize the domains and the Multifrontal Massively Parallel Sparse direct solver (MUMPS) was used to solve the heat transfer problem.

\section{References}

(1) Elert, G. The physics hypertextbook. Found July 1998, 9, 2008.

(2) Johnson, P. B.; Christy, R.-W. Optical constants of the noble metals. Physical review B $\mathbf{1 9 7 2 , 6 , 4 3 7 0 .}$ 
(3) Johnson, S. G. Notes on perfectly matched layers (PMLs). Lecture notes, Massachusetts Institute of Technology, Massachusetts 2008, 29.

(4) Berenger, J.-P. A perfectly matched layer for the absorption of electromagnetic waves. Journal of computational physics 1994, 114, 185-200.

(5) Rappaport, C. M. Perfectly matched absorbing boundary conditions based on anisotropic lossy mapping of space. IEEE Microwave and Guided Wave Letters 1995, 5, 90-92.

(6) Sacks, Z. S.; Kingsland, D. M.; Lee, R.; Lee, J.-F. A perfectly matched anisotropic absorber for use as an absorbing boundary condition. IEEE transactions on Antennas and Propagation 1995, 43, 1460-1463.

(7) Teixeira, F. L.; Chew, W. C. General closed-form PML constitutive tensors to match arbitrary bianisotropic and dispersive linear media. IEEE Microwave and Guided Wave Letters 1998, 8, 223-225. 